\title{
含有螺环己烷结构的稠环斯德酮的合成
}

\author{
张站斌* 张红海 \\ (北京师范大学化学学院 北京 100875)
}

摘要 3-(3-芳基-3-羟基丙基)斯德酮在三氟化嗍乙醚的催化作用下可以与环己酮发生类似于 Oxa-Pictet-Spengler 反应的 成环反应，生成带有螺环己烷结构的稠环斯德酮.

关键词 斯德酮; 环己酮; 螺环化合物; 合成

\section{Synthesis of Fused-Ring Sydnones with a Spirocyclohexane Moiety}

\author{
Zhang, Zhanbin* Zhang, Honghai \\ (College of Chemistry, Beijng Normal University, Beijing 100875)
}

\begin{abstract}
Fused-ring sydnones with a spiro cyclohexane moiety were prepared via oxa-Pictet-Spengler reaction of 3-(3-aryl-3-hydroxypropyl)sydnones with cyclohexanone in the presence of boron trifluoride ether complex.

Keywords sydnone; cyclohexanone; spiro compound; synthesis
\end{abstract}

斯德酮是 1935 年由 Earl 和 Mackney 发现的具有介 离子结构的五元杂环化合物, 其结构特殊, 可用结构式 1 表示 ${ }^{[1]}$. 由于其结构的特殊性以及某些斯德酮衍生物 具有生物和药物活性而引起人们的兴趣 ${ }^{[2]}$. 斯德酮环具 有芳香性，4-位无取代基的斯德酮可以发生亲电取代反 应 $^{[3]}$ 、Mannich 反应 ${ }^{[4]}$ 、金属化反应以及偶联反应 ${ }^{[5]}$ 等; 同 时斯德酮环是一个 1,3 -偶极体系, 可以发生 1,3-偶极环 加成反应 ${ }^{[6]}$. 斯德酮与炔烃进行环加成反应可以得到吡 唑衍生物 ${ }^{[7]}$, 是一个十分有用的合成吡唑的前体. 因此 合成新渘结构的斯德酮具有一定的意义.

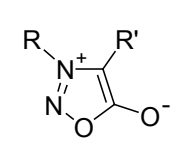

1

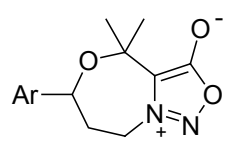

2

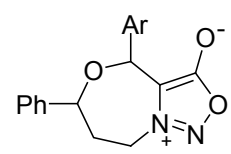

3
在以前的工作中, 我们开展了利用开链酮 $(\text { 丙酮 })^{[8]}$ 及芳香醛 ${ }^{[9]}$ 与 3-(3-芳基-3-差基丙基)斯德酮的 oxa-Pictet-Spengler 反应合成稠环斯德酮 2 和 3. 本文将报道环 酮与 3-(3-芳基-3-羊基丙基)斯德酮在三氟化嗍乙醚催化 下发生 oxa-Pictet-Spengler 反应, 并合成带有螺环结构 单元的稠环斯德酮化合物 $\mathbf{5 a} \sim \mathbf{5 g}$ (Eq. 1).

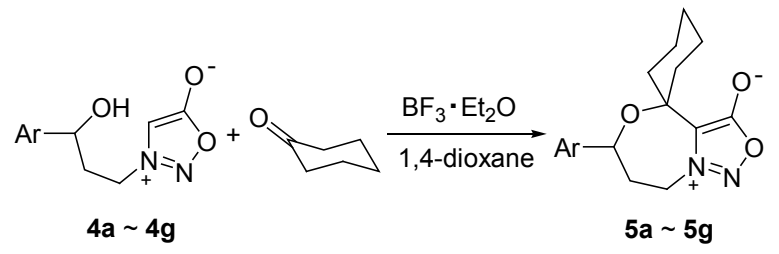

a: $\mathrm{Ar}=\mathrm{C}_{6} \mathrm{H}_{5} ; \mathbf{b}: \mathrm{Ar}=p-\mathrm{ClC}_{6} \mathrm{H}_{4} ; \mathbf{c}: \mathrm{Ar}=p-\mathrm{BrC}_{6} \mathrm{H}_{4} ; \mathbf{d}: \mathrm{Ar}=p-\mathrm{MeC}_{6} \mathrm{H}_{4}$;

e: $\mathrm{Ar}=p-\mathrm{O}_{2} \mathrm{NC}_{6} \mathrm{H}_{4} ; \mathbf{f}: \mathrm{Ar}=$<smiles></smiles>

\section{1 实验部分}

\section{1 仪器与试剂}

红外光谱使用 AVATAR 360 FT-IR 红外光谱仪(溴 化钾压片)测定; 核磁共振谱使用 Bruker AV 500 型超导 核磁共振仪测定, TMS 为内标; 质谱用 TRACE MS 型质 谱仪测定; 元素分析用 elementer Vario EL 型元素分析 仪测定.

所用试剂除特别注明外，均为市售分析纯或化学纯 商品.

\subsection{3-(3-芳基-3-羟基丙基)斯德酮 $4 \mathrm{a} \sim 4 \mathrm{~g}$ 的合成}

参考文献[10], 在冰水浴冷却下, 将 $\mathrm{NaBH}_{4}(0.1 \mathrm{~g}$,

*E-mail: zhangzb@bnu.edu.cn

Received April 8, 2011; revised September 19, 2011; accepted October 25, 2011. 
$2.6 \mathrm{mmol}$ )加入到 3-芳酰基乙基斯德酮的乙醇 $(10 \mathrm{~mL}$ )溶 液中, 继续搅拌 $1 \mathrm{~h}$. 将反应混合物倒入水中, 有固体析 出, 抽滤得产物, 用 $95 \%$ 乙醇重结晶得到纯品.

\subsection{3-(3-芳基-3-羟基丙基)斯德酮与环己酮的反应制 备化合物 $5 \mathrm{a} \sim 5 \mathrm{~g}$}

将 3-(3-芳基-3-羊基丙基)斯德酮(1.5 mmol)与环已 酮(3.0 mmol)溶于 1,4-二氧六环 $(10 \mathrm{~mL})$ 中, 在冰水浴冷 却下滴入 $\mathrm{BF}_{3} \cdot \mathrm{Et}_{2} \mathrm{O}$ 溶液 $(0.5 \mathrm{~mL})$, 混合液颜色逐渐变深, 室温放置 $1 \mathrm{~d}$, 再滴加 $\mathrm{BF}_{3} \cdot \mathrm{Et}_{2} \mathrm{O}$ 溶液 $(0.5 \mathrm{~mL})$. 用薄层色 谱跟踪反应, 至原料完全消失. 将混合液倒入分液漏斗, 用饱和 $\mathrm{NaHCO}_{3}$ 溶液洗涤处理, 用乙酸乙酯萃取, 乙酸 乙酯层再用饱和 $\mathrm{NaHCO}_{3}$ 溶液洗涤 2 次, 用无水 $\mathrm{NaSO}_{4}$ 干燥后, 旋转蒸发除去乙酸乙酯, 用柱层析分离得到产 品.

5a: 产率 43\%, m.p. $184 \sim 185{ }^{\circ} \mathrm{C} ;{ }^{1} \mathrm{H} \mathrm{NMR}\left(\mathrm{CDCl}_{3}\right.$, $500 \mathrm{MHz}) \delta: 1.29 \sim 2.59(\mathrm{~m}, 12 \mathrm{H}), 4.45(\mathrm{ddd}, J=13.2$, $4.4,3.0 \mathrm{~Hz}, 1 \mathrm{H}), 4.74 \sim 4.80(\mathrm{~m}, 1 \mathrm{H}), 4.85(\mathrm{dd}, J=8.8,6.0$ $\mathrm{Hz}, 1 \mathrm{H}), 7.29 \sim 7.42(\mathrm{~m}, 5 \mathrm{H}) ;{ }^{13} \mathrm{C} \mathrm{NMR}\left(\mathrm{CDCl}_{3}, 125\right.$ $\mathrm{MHz}) \delta: 166.81,141.85,128.72,127.72,125.27,110.22$, $76.82,70.73,49.43,36.44,35.18,29.14,24.59,21.09$, 20.83; IR (KBr) v: 2932, 1745, 1507, 1074, 1014, 812 $\mathrm{cm}^{-1}$; MS (EI) m/z: $301\left(\mathrm{M}^{+}+1\right), 300\left(\mathrm{M}^{+}\right)$. Anal. calcd for $\mathrm{C}_{17} \mathrm{H}_{20} \mathrm{~N}_{2} \mathrm{O}_{3}$ : C 67.98, H 6.71, N 9.33; found $\mathrm{C} 68.11$, H 6.81, N 9.26.

5b: 产率 51\%, m.p. 203 204 ${ }^{\circ} \mathrm{C} ;{ }^{1} \mathrm{H} \mathrm{NMR}\left(\mathrm{CDCl}_{3}\right.$, $500 \mathrm{MHz}) \delta: 1.32 \sim 2.58(\mathrm{~m}, 12 \mathrm{H}), 4.45(\mathrm{ddd}, J=13.3$, 4.7, 3.0 Hz, 1H), 4.75 (dt, $J=13.0,4.9 \mathrm{~Hz}, 1 \mathrm{H}), 4.82$ (dd, $J=8.9,6.0 \mathrm{~Hz}, 1 \mathrm{H}), 7.34 \sim 7.39$ (m, 4H, ArH); ${ }^{13} \mathrm{C} \mathrm{NMR}$ $\left(\mathrm{CDCl}_{3}, 125 \mathrm{MHz}\right) \delta: 20.83,21.08,24.52,29.09,35.12$, 36.40, 49.32, 70.21, 77.02, 109.99, 126.66, 128.93, 133.54, 140.31, 166.71; IR (KBr) v: 2928, 1747, 1492, 1185, 1015, $824 \mathrm{~cm}^{-1}$; MS (EI) m/z: $334\left(\mathrm{M}^{+}\right), 335\left(\mathrm{M}^{+}+1\right)$. Anal. calcd for $\mathrm{C}_{17} \mathrm{H}_{19} \mathrm{~N}_{2} \mathrm{O}_{3} \mathrm{Cl}$ : C 60.99, $\mathrm{H}$ 5.72, $\mathrm{N}$ 8.37; found $\mathrm{C}$ 61.11, H 5.92, N 8.31.

5c: 产率 $40 \%$, m.p. $212 \sim 213{ }^{\circ} \mathrm{C} ;{ }^{1} \mathrm{H}$ NMR $\left(\mathrm{CDCl}_{3}\right.$, $500 \mathrm{MHz}) \delta: 1.30 \sim 2.58(\mathrm{~m}, 12 \mathrm{H}), 4.72 \sim 4.82(\mathrm{~m}, 1 \mathrm{H})$, $4.43 \sim 4.47(\mathrm{~m}, 1 \mathrm{H}), 4.81(\mathrm{dd}, J=8.9,6.1 \mathrm{~Hz}, 1 \mathrm{H}), 7.30(\mathrm{~d}$, $J=8.6 \mathrm{~Hz}, 2 \mathrm{H}), 7.54(\mathrm{~d}, J=8.3 \mathrm{~Hz}, 2 \mathrm{H}) ;{ }^{13} \mathrm{C} \mathrm{NMR}$ $\left(\mathrm{CDCl}_{3}, 125 \mathrm{MHz}\right) \delta: 20.84,21.09,24.52,29.08,35.07$, 36.40, 49.31, 70.24, 77.04, 109.98, 121.63, 126.99, 131.89, 140.83, 166.70; IR (KBr) v: 2933, 1746, 1501, 1073, 1015, $816 \mathrm{~cm}^{-1}$; MS (EI) m/z: $378\left(\mathrm{M}^{+}\right) 379\left(\mathrm{M}^{+}+1\right)$. Anal. calcd for $\mathrm{C}_{17} \mathrm{H}_{19} \mathrm{~N}_{2} \mathrm{O}_{3} \mathrm{Br}$ : C 53.84, H 5.05, N 7.39; found $\mathrm{C}$ 54.02, H 5.20, N 7.45.

5d: 产率 36\%, m.p. $191 \sim 192{ }^{\circ} \mathrm{C} ;{ }^{1} \mathrm{H} \mathrm{NMR}\left(\mathrm{CDCl}_{3}\right.$,
$500 \mathrm{MHz}) \delta: 1.32 \sim 2.55(\mathrm{~m}, 12 \mathrm{H}), 2.39$ (s, 3H), 4.77 (ddd, $J=13.01,11.66,4.83 \mathrm{~Hz}, 1 \mathrm{H}), 4.82(\mathrm{dd}, J=8.9,6.1 \mathrm{~Hz}$, $1 \mathrm{H}), 7.22$ (d, $J=7.9 \mathrm{~Hz}, 2 \mathrm{H}), 7.30(\mathrm{~d}, J=8.0 \mathrm{~Hz}, 2 \mathrm{H}) ;{ }^{13} \mathrm{C}$ NMR $\left(\mathrm{CDCl}_{3}, 125 \mathrm{MHz}\right) \delta: 20.84,21.07,21.14,24.60$, $29.17,35.17,36.42,49.44,70.68,76.76,110.27,125.26$, 129.37, 137.46, 138.87, 166.82; IR (KBr) v: 2935, 1748, 1507, 1186, 1017, $815 \mathrm{~cm}^{-1}$; MS (EI) $m / z: 315\left(\mathrm{M}^{+}\right)$. Anal. calcd for $\mathrm{C}_{18} \mathrm{H}_{22} \mathrm{~N}_{2} \mathrm{O}_{3}$ : C 68.77, $\mathrm{H} \mathrm{7.05,} \mathrm{N} \mathrm{8.91;} \mathrm{found} \mathrm{C}$ 68.91, H 7.14, N 8.98.

5e: 产率 35\%, m.p. $223 \sim 224{ }^{\circ} \mathrm{C} ;{ }^{1} \mathrm{H} \mathrm{NMR}\left(\mathrm{CDCl}_{3}\right.$, $500 \mathrm{MHz}) \delta: 1.68 \sim 2.67(\mathrm{~m}, 12 \mathrm{H}), 4.50(\mathrm{ddd}, J=13.3$, $4.9,2.9 \mathrm{~Hz}, 1 \mathrm{H}), 4.78$ (ddd, $J=13.2,11.7,4.9 \mathrm{~Hz}, 1 \mathrm{H})$, $4.96(\mathrm{dd}, J=9.2,6.1 \mathrm{~Hz}, 1 \mathrm{H}), 7.62(\mathrm{~d}, J=8.7 \mathrm{~Hz}, 2 \mathrm{H})$, $8.29(\mathrm{~d}, J=8.7 \mathrm{~Hz}, 2 \mathrm{H}) ;{ }^{13} \mathrm{C}$ NMR $\left(\mathrm{CDCl}_{3}, 125 \mathrm{MHz}\right) \delta$ : 20.89, 21.13, 24.44, 28.98, 34.97, 36.43, 49.18, 70.01, $77.45,109.65,124.13,126.08,147.51,148.84,166.58$; IR (KBr) v: 2941, 1728, 1518, 1350, 1087, 1003, $851 \mathrm{~cm}^{-1}$; MS (EI) $m / z$ : $345\left(\mathrm{M}^{+}\right), 346\left(\mathrm{M}^{+}+1\right)$. Anal. calcd for $\mathrm{C}_{17} \mathrm{H}_{19} \mathrm{~N}_{3} \mathrm{O}_{5}$ : C 59.12, H 5.55, N 12.17; found C 59.38, H 5.76, N 12.13 .

5f: 产率 $33 \%$, m.p. $186 \sim 187{ }^{\circ} \mathrm{C} ;{ }^{1} \mathrm{H} \mathrm{NMR}\left(\mathrm{CDCl}_{3}\right.$, $500 \mathrm{MHz}) \delta: 1.28 \sim 2.82(\mathrm{~m}, 12 \mathrm{H}), 4.46(\mathrm{ddd}, J=13.1$, 4.6, 2.6 Hz, 1H), 4.89 (dt, $J=12.7,4.7 \mathrm{~Hz}, 1 \mathrm{H}), 5.49$ (dd, $J=7.6,7.2 \mathrm{~Hz}, 1 \mathrm{H}), 7.54 \sim 7.96(\mathrm{~m}, 7 \mathrm{H}) ;{ }^{13} \mathrm{C}$ NMR $\left(\mathrm{CDCl}_{3}, 125 \mathrm{MHz}\right) \delta: 20.85,21.20,24.56,29.05,34.76$, $36.65,49.40,68.75,77.25,110.21,122.99,123.18,125.69$, $125.77,126.40,128.31,129.24,129.36,133.94,137.65$, 166.87; IR (KBr) v: 2942, 1735, 1506, 1073, 1015, 817 $\mathrm{cm}^{-1}$; $\mathrm{MS}$ (EI) $m / z: 351\left(\mathrm{M}^{+}+1\right)$. Anal. calcd for $\mathrm{C}_{21} \mathrm{H}_{22} \mathrm{~N}_{2} \mathrm{O}_{3}$ : C 71.98, H 6.33, N 7.99; found C 72.10, H 6.31, N 8.03.

5g: 产率 $37 \%$, m.p. $171 \sim 172{ }^{\circ} \mathrm{C} ;{ }^{1} \mathrm{H}$ NMR $\left(\mathrm{CDCl}_{3}\right.$, $500 \mathrm{MHz}) \delta: 1.30 \sim 2.65(\mathrm{~m}, 12 \mathrm{H}), 4.45 \sim 4.49(\mathrm{~m}, 1 \mathrm{H})$, $4.79 \sim 4.85(\mathrm{~m}, 1 \mathrm{H}), 5.01(\mathrm{dd}, J=8.6,6.2 \mathrm{~Hz}, 1 \mathrm{H}), 7.51 \sim$ $7.92(\mathrm{~m}, 7 \mathrm{H}) ;{ }^{13} \mathrm{C} \mathrm{NMR}\left(\mathrm{CDCl}_{3}, 125 \mathrm{MHz}\right) \delta: 20.88$, 21.16, 24.61, 29.22, 35.12, 36.46, 49.49, 70.93, 76.93, $110.25,123.45,124.05,126.19,126.48,127.77,128.03$, $128.69,132.91,133.24,139.18,166.83$; IR (KBr) v: 2946, 1720, 1507, 1074, 1014, $813 \mathrm{~cm}^{-1}$; MS (EI) $\mathrm{m} / z$ : 351 $\left(\mathrm{M}^{+}+1\right)$. Anal. calcd for $\mathrm{C}_{21} \mathrm{H}_{22} \mathrm{~N}_{2} \mathrm{O}_{3}: \mathrm{C} 71.98, \mathrm{H} 6.33, \mathrm{~N}$ 7.99; found C 71.99, H 6.26, N 8.31.

\section{2 结果与讨论}

\section{1 反应条件}

3-(3-苯基-3-差基丙基)斯德酮与芳香醛的反应是在 
二氯甲烷中用三氟化硼乙醚作催化剂进行的, 但是在相 同条件下 3-(3-苯基-3-羊基丙基)斯德酮与环己酮的反应 时, 得到一个非常复杂的化合物, 无法分离得到目标产 物. 当改用 1,4-二氧六环做溶剂后, 反应可以顺利进行, 得到目标产物; 但是如果将催化剂三氟化硼乙醚一次性 加入体系, 生成较多的副产物, 产率很低; 因此将催化 剂三氟化嗍乙醚分批加入反应体系, 减少了副产物, 提 高了产率.

\section{2 与其它环酮的反应}

3-(3-芳基-3-羊基丙基)斯德酮与环戊酮、环庚酮无 论是在二氯甲烷中还是 $1,4-$ 二氧六环均不发生类似于环 己酮的反应.

\section{3 化合物 $\mathbf{5}$ 的波谱性质}

3-(3-芳基-3-羊基丙基)斯德酮分子中斯德酮环的 4位没有取代基, 故其 ${ }^{1} \mathrm{H}$ NMR 谱图中于 $\delta 5.5$ 左右有 4位氢核的特征吸收峰，当 3-(3-芳基-3-羊基丙基)斯德酮 与环已酮反应生成具有螺环已烷结构的稠环斯德酮后, 在 ${ }^{1} \mathrm{H}$ NMR 谱图中位于 $\delta 5.5$ 左右的峰没有了, 而在 $\delta$ 1.3 2.6 之间出现了螺环已烷上的五个亚甲基氢核的吸 收峰; 在 ${ }^{13} \mathrm{C} N M R$ 谱图中, 于 $\delta 20 \sim 50$ 之间出现了 6 个 螺环已烷的碳原子吸收峰, 证明生成了具有螺环已烷结 构的稠环斯德酮.

\section{4 化合物 $5 f$ 的 $X$ 射线单晶衍射分析}

为了进一步确定目标化合物的结构, 我们对化合物 $\mathbf{5 f}$ 进行了 $\mathrm{X}$ 射线单晶衍射分析, 数据存于英国剑桥数据 中心, $\mathrm{CCDC}$ 号为 862450 . 由化合物 $\mathbf{5 f}$ 的 X 射线单晶衍 射结构图(图 1)可以看出螺环己烷结构的存在. 化合物 $\mathbf{5 f}$ 的单晶衍射数据是在 Bruker Smart CCD 衍射仪上, 采 用经石墨单色化的 Mo Ka 射线 $(\lambda=0.71073 \AA)$, 在 $294 \mathrm{~K}$ 温度下收集的. 吸收校正用 SADABS 程序, 晶体结构修 正用 SHEXLX-97 程序, 氢原子坐标由差值 Fourier 合成 法得到. 分子式为 $\mathrm{C}_{21} \mathrm{H}_{22} \mathrm{~N}_{2} \mathrm{O}_{3}$, 分子量为 350.41 , 该晶 体属于单斜晶系, 空间群为 $P 2_{1} / c$, 晶胞参数 $a=$ 10.203(3) $\AA, b=9.415(2) \AA, c=19.152(5) \AA, \alpha=90^{\circ}, \beta=$ 104.677(4) $)^{\circ}, \gamma=90^{\circ}, V=1779.8(8) \AA^{3}, Z=4, F(000)=$ $744, \mu=0.088 \mathrm{~mm}^{-1}, R_{\mathrm{int}}=0.0343, R\left[F^{2}>2 \sigma\left(F^{2}\right)\right]=0.040$, $w R\left(F^{2}\right)=0.0876$.

\section{3 结果与讨论}

在三氟化嗍乙醚的催化作用下, 3-(3-芳基-3-差基丙

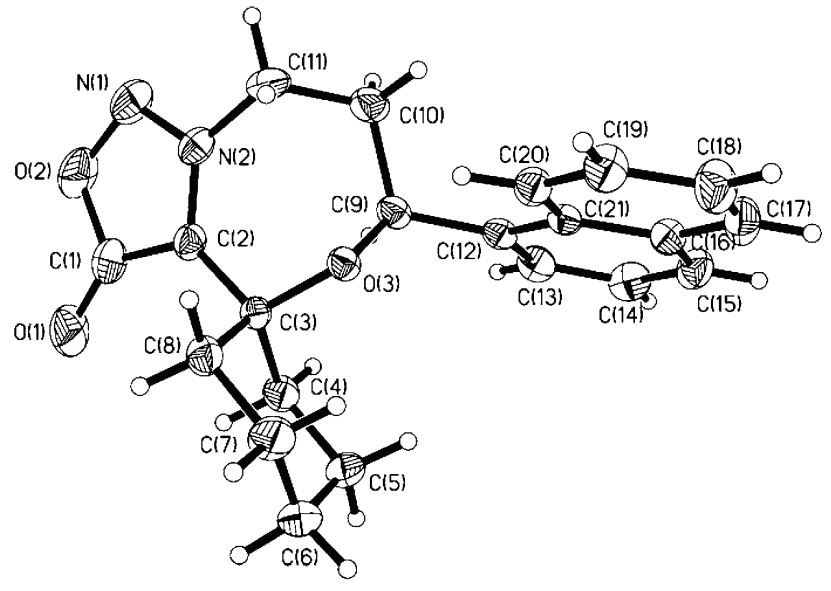

图 1 化合物 $\mathbf{5 f}$ 的晶体结构图

Figure 1 Crystal structure of compound $\mathbf{5 f}$

基)斯德酮与环己酮在 1,4-二氧六环中反应，得到具有螺 环已烷结构的稠环斯德酮, 所得化合物的结构经过 ${ }^{1} \mathrm{H}$ $\mathrm{NMR},{ }^{13} \mathrm{C}$ NMR, MS, IR，元素分析的鉴定，化合物 $\mathbf{5 f}$ 的 结构经 X 射线单晶衍射进一步验证. 在相同条件下，环 戊酮和环庚酮不发生类似反应.

\section{References}

[1] Earl, J. C.; Mackney, A. W. J. Chem. Soc. 1935, 899.

[2] (a) Browne, D. L.; Harrity, J. P. A. Tetrahedron 2010, 66, 553. (b) Stewart, F. H. C. Chem. Rev. 1964, 64, 129.

(c) Kawase, M.; Sakagami, H.; Motohashi, N. Top. Heterocycl. Chem. 2009, 16, 135.

[3] Srivastava, K. P.; Mishra, P. K.; Kumari, S. Rasayan J. Chem. 2010, 3,140 .

[4] Zhang, Z.-B.; Yin, C.-L.; Wu, Y.-R. Chin. J. Org. Chem. 2002, 22, 283 (in Chinese). (张站斌, 尹承烈, 吴永仁, 有机化学, 2002, 22, 283.)

[5] (a) Delaunay, T.; Genix, P.; Es-Sayed, M.; Vors, J.-P.; Monteiro, N.; Balme, G. Org. Lett. 2010, 12, 3328.

(b) Rodriguez, A.; Moran, W. J. Synthesis 2009, 650.

[6] Wu, C.; Fang, Y.; Larock, R. C.; Shi, F. Org. Lett. 2010, 12, 2234.

[7] (a) Browne, D. L.; Taylor, J. B.; Plant, A.; Harrity, J. P. A. J. Org. Chem. 2010, 75, 984.

(b) Browne, D. L.; Taylor, J. B.; Plant, A.; Harrity, J. P. A. J. Org. Chem. 2009, 74, 396.

[8] Zhang, Z. B.; Wu, Y. R.; Yin, C. L. Synth. Commun. 2002, 32, 2203.

[9] Zhang, Z.; Duan, X. Heterocycles 2005, 65, 2649.

[10] Zhang, Z.; Zhang, R.; Yin, C.; Wu, Y. J. Beijing Normal Univ. (Nat. Sci.) 1997, 33, 521 (in Chinese).

(张站斌; 张瑞德, 尹承烈, 吴永仁, 北京师范大学学报(自然科 学版), 1997, 33, 521.)

(Zhao, C.; Fan, Y.) 